\title{
LETTER FROM THE EDITOR IN CHIEF
}

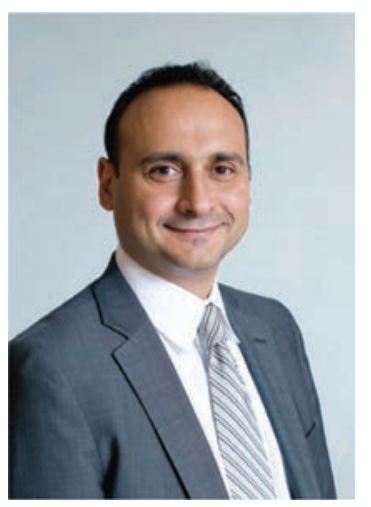

Dear Readers,

This issue of the Journal contains many interesting publications. I would like to highlight two articles describing performing ablation without the use of fluoroscopy. In the first article, Gautam et al. report a series of 20 patients who underwent flutter ablation using 3D electroanatomic mapping without the use of fluoroscopy. In the second article, Montgomery et al. describe zero-fluoroscopy, completely ICE-guided approach to ablation of atrial fibrillation using a single transseptal access, single force-sensing radiofrequency ablation catheter, and a coronary sinus decapolar catheter. In both studies, the procedures were completed successfully and there were no complications.

Over the past decade, many reports have demonstrated that patients are being exposed to higher doses of ionizing radiation secondary to cardiac diagnostic and therapeutic procedures. These include among others, coronary intervention, catheter ablation for arrhythmias, and imaging. This increased exposure has resulted in a higher incidence of cancer in patients undergoing these procedures. More interestingly, there were studies also reporting an increased incidence of left-sided brain cancer among cardiologists and radiologists performing these procedures. These concerning findings, in addition to the musculoskeletal effects of chronic use of lead apron, have fueled a strong interest for the development of new technologies that help reduce the exposure to radiation. New electroanatomical mapping systems that are integrated with fluoroscopy have been developed and allow performing catheter ablation and cardiac resynchronization procedures with minimal radiation use. These new technologies and others are expected to help significantly reduce radiation exposure to both patient and physician.

This issue of the Journal also marks the end of the year 2015. This has been a very fascinating and productive year for the field of cardiac electrophysiology. On the ablation front the interest in contact force sensing technology grew rapidly over the past year. The result of the LASER balloon randomized study showing non-inferiority to radiofrequency ablation for the treatment of paroxysmal atrial fibrillation was also reported in 2015. The debate over the role of rotors in the maintenance of atrial fibrillation persisted for the whole year. On the device front, the interest in MRI compatible pacemakers and ICDs continued to grow. It is expected that these areas will keep on generating significant attention in 2016.

I hope that you enjoy reading this issue of the Journal. Best wishes for a happy and healthy New Year.

Regards,

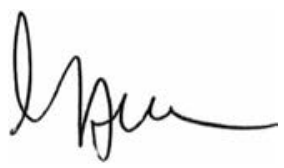

Moussa Mansour, MD, FHRS, FACC

Editor-in-Chief

The Journal of Innovations in Cardiac Rhythm Management

MMansour@InnovationsInCRM.com

Director, Cardiac Electrophysiology Laboratory

Director, Atrial Fibrillation Program

Massachusetts General Hospital

Boston, MA 